\title{
Perineal Groove in a Neonate With Increased Maternal Serum Alpha-Fetoprotein
}

\author{
Jameson M. Petrochko ${ }^{\mathrm{a}, \mathrm{b}}$, Jennifer A. Canning ${ }^{\mathrm{c}}$, Chaminie A. Wheeler ${ }^{\mathrm{c}, \mathrm{d}, \mathrm{e}, \mathrm{f}}$
}

\begin{abstract}
Perineal groove is a likely underreported congenital defect that usually occurs in females, characterized by the presence of a sulcus coursing between the genitalia and the anus. We present the case of a neonate with this defect and discuss its clinical implications and relationship to the mother's sequential screen.
\end{abstract}

Keywords: Perineal groove; Congenital defect; Neonate; MSAFP

\section{Introduction}

Perineal groove is essentially a longitudinal sulcus in the perineum extending anteriorly from the anus towards the vagina. The incidence is unclear, but it is likely underreported in the literature [1]. The lack of awareness that leads to underreporting also makes the diagnosis challenging, and perineal groove is often initially misdiagnosed. It is an overwhelmingly female phenomenon: only one infant out of the 40 cases found in the literature was male, although reports exist of other cases [2]. The original description of the abnormality by Stephens [3] consists of three aspects: a wet sulcus between the fourchette and the anus, hypertrophied minoral tails leading to the anus, and a normal vestibule. Its embryologic origin is unclear, as is the origin of a normal perineum. Interestingly, the mother of our patient had an increased maternal serum alpha-fetoprotein

Manuscript submitted June 9, 2020, accepted July 15, 2020

Published online November 3, 2020

${ }^{a}$ Lewis Katz School of Medicine at Temple University, Philadelphia, PA, USA ${ }^{b}$ Department of General Surgery, St. Luke's University Health Network, Bethlehem, PA, USA

'St. Luke's University Health Network, Anderson Campus, 1872 St. Luke's Boulevard Easton, PA, USA

dDivision of Neonatology, Department of Pediatrics, St. Luke's University Hospital Network, Bethlehem, PA, USA

eDepartment of Pediatrics, Lewis Katz School of Medicine at Temple University, Philadelphia, PA, USA

${ }^{f}$ Corresponding Author: Chaminie A. Wheeler, St. Luke's University Health Network, 801 Ostrum Street, Bethlehem, PA 18015, USA.

Email: CWheeler@onsiteneonatal.com

doi: https://doi.org/10.14740/ijcp383
(MSAFP) level on sequential screen which is associated with other open congenital defects. Here we present the case of a female neonate with a defect consistent with the aforementioned triad. We discuss clinical pearls of this abnormality as well as a possible relationship between its embryology and the mother's abnormal sequential screen.

\section{Case Report}

Baby girl X was a 3,827 g appropriate-for-gestational-age (AGA) neonate born at gestational age of 41 weeks and 4 days. The infant's mother was a 33-year-old primigravida Caucasian woman who received adequate prenatal care. Maternal medical history was significant for anemia (no prepregnancy hemoglobin, 10.4 just prior to delivery), and obesity (body mass index (BMI) 29.31 year prior to conception, 32.4 at 12 weeks and 5 days). Maternal allergies included cats (shortness of breath), fruits (facial swelling), and seasonal rhinitis. Medications included cyclobenzaprine (flexeril, prescribed at 37 weeks for back spasms), ferrous sulfate, prenatal multivitamin, and aspirin (adherence unclear). She denied use of tobacco, alcohol, and recreational drugs throughout pregnancy. Maternal ancestry was German/Irish, and family history was significant for pancreatic, colon, and ovarian cancer in the maternal grandfather, maternal grandmother, and mother, respectively. There is no maternal family history of congenital abnormalities, including midline defects. Paternal ancestry was German although paternal family and medical history were unavailable. Consanguinity status was unknown. Prenatal labs were significant only for an abnormal stepwise sequential screen (Table 1). The abnormal sequential screen was managed with aspirin to minimize risk of pre-eclampsia (adherence unclear), and anatomical ultrasounds at 28, 32, and 36 weeks. The only abnormal finding was uterine synechiae, discovered at 37 weeks and 1 day, which did not inhibit fetal movement.

Delivery was induced, as scheduled, due to post-dates, and rupture of membranes was prolonged $(25 \mathrm{~h}$ and $11 \mathrm{~min})$. The infant was delivered via low transverse cesarean section due to failure to progress and was found to be in brow presentation. Appearance, Pulse, Grimace, Activity, and Respiration (APGAR) scores were 9 at $1 \mathrm{~min}$ and 9 at $5 \mathrm{~min}$ (-1 for color for both). The infant's vital signs at birth were 98.8 degrees Fahrenheit, heart rate 150 beats per minute, and respirations 60 per minute. Vitals remained stable throughout the admis- 
Table 1. Results of Stepwise Sequential Screen

\begin{tabular}{lll} 
Step & Test $(\mathbf{G A})$ & Value \\
\hline 1 & PAPP-A (13 weeks and 1 day by dates) & $0.20 \mathrm{MoM}(\downarrow)$ \\
& Beta-hCG (13 weeks and 1 day by dates) & $0.41 \mathrm{MoM}$ \\
2 & NT (12 weeks and 5 days by dates, 12 weeks and 3 days by CRL) & $0.78 \mathrm{MoM}$ \\
& MSAFP (17 weeks and 1 day by dates) & $3.12 \mathrm{MoM}(\uparrow)$ \\
& Beta-hCG (17 weeks and 1 day by dates) & $1.26 \mathrm{MoM}$ \\
& Estriol (17 weeks and 1 day by dates) & $1.31 \mathrm{MoM}$ \\
& Inhibin-A (17 weeks and 1 day by dates) & $2.13 \mathrm{MoM}(\uparrow)$ \\
\hline
\end{tabular}

PAPP-A: pregnancy-associated plasma protein A; MoM: multiples of median; beta-hCG: beta human chorionic gonadotropin; NT: nuchal translucency; CRL: crown rump length; MSAFP: maternal serum alpha-fetoprotein; GA: gestational age.

sion except for two abnormal axillary temperatures of 97.6 and 97.2 degrees Fahrenheit at $26 \mathrm{~h}$ and $48 \mathrm{~h}$ of life, respectively. Both low temperatures resolved after swaddling the infant in a heated blanket. Glucose remained within normal limits. The infant voided and passed stool appropriately.

On physical exam, the infant was found to have caput succedaneum and a perineal groove. The genitourinary exam showed otherwise normal female genitalia and a patent vagina and anus. The perineal groove was a $1 \mathrm{~mm}$ wide $\times 1 \mathrm{~mm}$ deep, erythematous, wet sulcus running from the posterior fourchette of the vagina to the anus (Fig. 1). The groove is flanked by minoral tails, which are an extension of the labia minora and run to the anus. No fistulas, sinuses, or tracks were noted. There was no drainage, bleeding, discoloration, or signs of infection. The remainder of the physical exam was normal. On the second day of life, glucose was $58 \mathrm{mg} / \mathrm{dL}$ and total bilirubin was $3.33 \mathrm{mg} / \mathrm{dL}$ at $29 \mathrm{~h}$ of life.

The appearance of the groove did not change over the first $48 \mathrm{~h}$ of life. The parents were counseled that the groove would likely close spontaneously by 2 years of age. They were further educated regarding proper cleaning of the area, as the gap in the epithelium predisposes the area to infection from fecal bacteria. Critical congenital heart disease screen was normal with $\mathrm{SpO}_{2}$ of $98 \%$ in the right upper extremity and $100 \%$ in the left upper extremity. The hearing screening was normal. Discharge physical exam was normal except for the perineal groove (Table 2).

The perineal groove was identified by the pediatrician while conducting a well-child exam on the fourth day of life. The pediatrician consulted urology, pediatric surgery and plastic surgery. Pediatric urology, pediatric surgery, and plastic surgery agreed with the diagnosis of perineal groove and plan of care. The parents were counseled to keep the area clean and that the defect would likely spontaneously resolve. No other defects were noted on physical exam. In accordance with current (literature) recommendations, no additional testing for associated defects was performed. The patient experienced some intermittent erythema of the perineum possibly related to a zinc oxide ointment that ultimately resolved with an unspecified diaper cream. At 4 months of age the perineal groove was noted to be completely closed. The congenital disorder screen was normal (Table 3 ). The child has met all milestones, has been growing at a normal pace, and has been healthy aside from urticarial allergic reactions to egg and peanut butter, and an episode of viral gastroenteritis.

\section{Discussion}

\section{Perineal groove}

Our patient was a female neonate who presented asymptomatically while still in the newborn nursery. While female infants with a perineal groove occasionally have other associated urogenital or anorectal malformations [4-6] or other medical conditions [7], ours did not. The male case of perineal groove had the groove terminating at the urethra (located abnormally at the penoscrotal junction) and was associated with bifid scrotum, hypospadias, chordee, and fecal incontinence [2]. Rou-

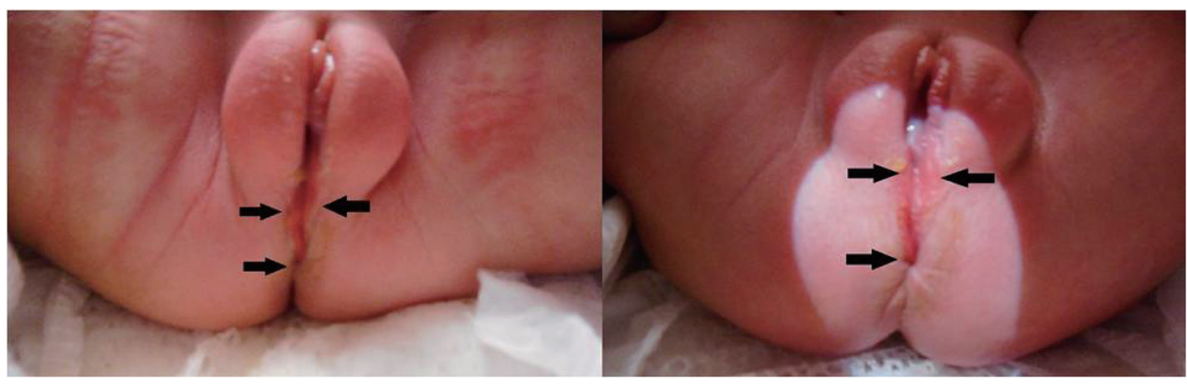

Figure 1. Perineal groove in a female neonate at 3 days of life. The anterior/superior aspect of the groove is formed by hypertrophied minoral tails (double arrows) and courses posteriorly/inferiorly to the anal verge (single arrows). 
Table 2. Discharge Physical Exam on the Third Day of Life, Normal Except for the Perineal Groove

\begin{tabular}{ll}
\hline General appearance & Alert, active, no distress \\
\hline Head & Normocephalic, anterior fontanel open and flat \\
Eyes & Conjunctiva clear, positive red reflex bilaterally \\
Ears & Normally placed, no anomalies \\
Nose & Nares patent \\
Mouth & Palate intact \\
Respiratory & Breath sounds clear and equal without grunting, flaring, or retractions \\
Cardiovascular & Regular rate and rhythm without murmur. Normal capillary refill, femoral pulses present \\
Abdomen & Soft, non-distended without masses or hepatosplenomegaly. Bowel sounds present \\
Genitourinary & Normal genitalia with perineal groove from labia to anus at 12 o'clock \\
Spine & No hair tufts or dimples \\
Musculoskeletal & Normal hips \\
Skin/hair/nails & Skin warm, dry, and intact without rashes \\
Neurologic & Normal tone and reflexes \\
\hline
\end{tabular}

tine screening of perineal groove patients for associated malformations is not necessary [8].

Embryologically, the endoderm gives rise to the lining of the digestive and respiratory systems, and part of the urogenital system. The ectoderm gives rise to the central nervous system and epidermis, and the mesoderm gives rise to the muscle and skeletal systems as well as parts of the urogenital system. The embryological origins of the perineum and perineal groove are not agreed upon, although mechanisms for perineal groove formation include persistence of the cloacal duct [4, 7-12], failure of cloacal fold fusion between the vestibule and anus $[3,6-11,13-15]$, or occurrence of a defect of it is a remnant of the urorectal septum (URS) $[9,11,16,17]$.

The perineal groove is often observed within the first few days of life. However, sometimes, it is initially misdiagnosed as other conditions, such as erosion [18], anal fissure [5], ulcerated hemangioma [19], infection [8, 13], or diaper dermatitis $[5,10,19]$. The differential also includes contact dermatitis [8], lichen sclerosis [8], perianal pyramidal protrusion [8], and trauma $[8,12]$. Failure of treatment, such as cortisone/gen-

Table 3. Results of the Pennsylvania Department of Health Mandatory Congenital Disorder Screening Plus Supplemental Newborn Screening

\begin{tabular}{lll}
\hline Target congenital disorder/primary analyte tested & Result & Reference range (for newborns) \\
\hline Congenital adrenal hyperplasia/17-OH progesterone & $5.0 \mathrm{ng} / \mathrm{mL}$ & $<25 \mathrm{ng} / \mathrm{mL}$ \\
Hypothyroidism/thyroxine & $19.4 \mu \mathrm{g} / \mathrm{dL}$ & $>6.0 \mu \mathrm{g} / \mathrm{dL}$ \\
Galactosemia/galactose & $2.5 \mathrm{mg} / \mathrm{dL}$ & $\geq 40 \mu \mathrm{M}$ \\
Galactosemia/uridyltransferase & $427.0 \mu \mathrm{dL}$ \\
Hemoglobinopathies/hemoglobin isoelectric focusing & $\mathrm{FA}$ & $\mathrm{FA}, \mathrm{AF}$ or $\mathrm{A}^{\mathrm{a}}$ \\
Maple syrup urine disorder/leucine & $\mathrm{WNL}$ & \\
MPS-1 & $\mathrm{WNL}$ & \\
Phenylketonuria/phenylalanine & $\mathrm{WNL}$ & \\
Pompe & $\mathrm{WNL}$ & \\
Spinal muscular atrophy & $\mathrm{WNL}$ & \\
X-linked adrenoleukodystrophy & $\mathrm{WNL}$ & \\
Acylcarnitine profile & $\mathrm{WNL}$ & \\
Amino acid profile & $\mathrm{WNL}$ & \\
Biotinidase deficiency & $48.0 \mathrm{ERU}$ & $\mathrm{WNL}$ \\
Cystic fibrosis/immunoreactive trypsinogen & $\mathrm{WNL}$ & $\mathrm{ERU}$ \\
G6PD DNA analysis & & \\
\hline
\end{tabular}

aCombinations of hemoglobin A and hemoglobin F. ERU: enzyme response unit; WNL: within normal limits. 
tamicin cream [18], barrier creams [5, 19], pulsed-dye laser [19], oral antihistamines [11], or antifungals [19], aimed at the incorrect diagnosis often prompts further investigation. At least one case was initially thought to be due to child sexual abuse [20]. Closer examination revealed regular margins, lack of granulation/healing, and a regular shape, all of which essentially eliminated sexual abuse from the differential diagnosis [21]. Proper documentation of the defect at the time of birth would help avoid misdiagnosis of sexual abuse [15]. Improvement of perineal grooves most often occurs over time, with improvement noted at 2 months [4], 1 year [14], 14 months [19], and 17 months [5] of life. The majority close spontaneously by 2 years of age [22]. Surgical intervention is usually not necessary, although indications include infection [8, 23], failure to spontaneously close [8], and cosmetic concern [16$18,23]$. Surgery in female patients tended to be successful, although the surgical sites were prone to dehiscence when not covered with surgical glue [23]. The male patient required several operations to correct all the abnormalities [2].

\section{Sequential screen}

The purpose of the sequential screen is to identify patients who might benefit from additional testing for aneuploidy or certain structural defects [24]. This patient's sequential screen results were not specifically consistent with any recognized disorder or structural defect. High inhibin-A is concerning for Down or Turner syndrome, which was not likely given the high MSAFP. Low pregnancy-associated plasma protein A (PAPP-A) is concerning for intrauterine growth restriction (IUGR) [25], which was not seen in our patient. Increased MSAFP is concerning for abnormal placentation [25-27] or a structural defect [28], specifically open defects, such as open neural tube, omphalocele, and gastroschisis [28]. However, none of this was observed on fetal ultrasound. Unexplained increased MSAFP is associated with an increased risk of adverse fetal outcomes including low birthweight, perinatal death [29], pre-term birth, and pre-eclampsia [30], none of which occurred.

Alpha-fetoprotein is produced by the yolk sac, fetal liver, and fetal gastrointestinal (GI) tract [26]. It enters maternal circulation via two mechanisms: transplacentally and from the amniotic membrane across fetal membranes [31]. In a normal pregnancy, MSAFP passes via urine from the fetus to the amniotic fluid, where levels of AFP remain lower than those in the fetal serum $[28,32]$. The dominant mechanism of AFP moving from fetal to maternal circulation is thought to be the transplacental route in healthy patients [31]. However, in a fetus with a neural tube defect, the AFP can leak from the fetal circulation to the amniotic fluid [29]. This effect likely is not limited to neural tube defects, however, and could apply to other open defects (e.g. gastroschisis, omphalocele). We hypothesize that the increased MSAFP is due to the open defect found in this patient. Although this patient's open defect is not as large as a gastroschisis or some neural tube defects, the high concentration gradient between fetal serum and amniotic fluid ( $>150: 1$ [32]) could contribute to the significant MSAFP. As discussed below, it is likely that the groove represents failure of epithelial closure, which would expose deeper mesodermal and endoder- mal tissue to the amniotic fluid.

\section{Conclusions}

Perineal groove might be a cause of increased MSAFP or other prenatal screening abnormalities in a pregnancy with a seemingly healthy fetus. Given its rarity and the unlikelihood of causing significant morbidity compared to other conditions associated with abnormal prenatal screening, little or no counseling is appropriate. It is, however, an excellent example of a benign possible etiology for the increased MSAFP.

These patients may have an increased risk for urinary tract infection (UTI), soft tissue infection due to lack of epithelialization and associated regional defects with consequence such as incontinence [13]. This defect tends to close on its own in the first few years of life, although surgical referral would be appropriate for recurrent infection, ulceration, failure to close spontaneously, and for associated significant anogenital defects. These other regional anatomic defects may be especially severe in male patients.

Clinicians should be aware of this anomaly so that unnecessary interventions and treatments for diaper rash, fungal infection, child abuse, or other causes can be avoided. The most appropriate management seems to be observation and extra attention to hygiene to prevent infection of this epithelial defect.

\section{Acknowledgments}

The authors would like to thank Austin Vitelli for valuable editorial assistance.

\section{Financial Disclosure}

None to declare.

\section{Conflict of Interest}

None to declare.

\section{Informed Consent}

The parents of baby girl X gave informed consent to review the medical records of baby girl $\mathrm{X}$ and her mother, and to publish a case report (including de-identified clinical images).

\section{Author Contributions}

JMP, JAC, and CAW participated in the conception and design of this work. The first draft was written by JMP. All authors critically evaluated the manuscript and provided feedback and have given final approval of this version to be published. All authors agree to be accountable for all aspects of this work. 


\section{Data Availability}

Any inquiries regarding supporting data availability of this study should be directed to the corresponding author.

\section{Abbreviations}

PAPP-A: pregnancy-associated plasma protein A; MoM: multiples of median; beta-hCG: beta human chorionic gonadotropin; NT: nuchal translucency; CRL: crown rump length; MSAFP: maternal serum alpha-fetoprotein; APGAR: Appearance, Pulse, Grimace, Activity and Respiration; URS: urorectal septum; IUGR: intrauterine growth restriction; GI: gastrointestinal; ERU: enzyme response unit; WNL: within normal limits; AGA: appropriate-for-gestational-age; BMI: body mass index; GA: gestational age; UTI: urinary tract infection

\section{References}

1. Sekaran P, Shawis R. Perineal groove: A rare congenital abnormality of failure of fusion of the perineal raphe and discussion of its embryological origin. Clin Anat. 2009;22(7):823-825.

2. Chatterjee SK, Chatterjee US, Chatterjee U. Perineal groove with penoscrotal hypospadias. Pediatr Surg Int. 2003;19(7):554-556.

3. Stephens FD. The female anus, perineum and vestibule. Embryogenesis and deformities. Aust N Z J Obstet Gynaecol. 1968;8(2):55-73.

4. Siruguppa K, Tuli SS, Kelly MN, Tuli SY. Newborn female with a midline perineal defect. Clin Pediatr (Phila). 2012;51(2):188-190.

5. Garcia-Palacios M, Mendez-Gallart R, Cortizo-Vazquez J, Rodriguez-Barca P, Estevez-Martinez E, Bautista-Casasnovas A. Perineal groove in female infants: A case series and literature review. Pediatr Dermatol. 2017;34(6):677680 .

6. Abdel Aleem A, el Sheikh S, Mokhtar A, Ghafouri H, Saleem M. The perineal groove and canal in males and females - a third look. Z Kinderchir. 1985;40(5):303-307.

7. Cheng H, Wang Z, Zhao Q, Zhu H, Xu T. Perineal groove: report of two cases and review of the literature. Front Pediatr. 2018;6:227.

8. Midline perineal lesion in a newborn girl. J Paediatr Child Health. 2018;54(7):814.

9. Jin ZW, Jin Y, Li XW, Murakami G, Rodriguez-Vazquez JF, Wilting J. Perineal raphe with special reference to its extension to the anus: a histological study using human fetuses. Anat Cell Biol. 2016;49(2):116-124.

10. Granillo Fernández S, Garone A, Molina LT, Luty G, Bello C, Ripoli M, Rolotti M, et al. Perineal groove: report of three cases. Journal of Dermatitis, 2018;3(11).

11. Tambe S, Kernland Lang K, Saple DG. Perineal groove: a rare anogenital anomaly. European Journal of Pediatric Dermatology. 2015;25:16-19.

12. Pereira C, Salgado M, Cascais M. Midline perineal lesion in a newborn girl. J Paediatr Child Health. 2018;54(7):813.

13. Harsono $\mathrm{M}$, Pourcyrous $\mathrm{M}$. Perineal groove: a rare congenital midline defect of perineum. AJP Rep. 2016;6(1):e30-32.

14. Kadowaki H, Nakahira M, Yamada C, Takeuchi S, Tamate S, Shiokawa C. Perineal groove and perineal canal. Jpn J Surg. 1983;13(3):216-218.

15. Butterfield RC, Iyengar S. A Newborn Girl with Unusual Genitalia. Pediatr Ann. 2016;45(1):e8-9.

16. Ramar P, Shringi S. Perineal groove: a rare and benign anorectal malformation. Journal of Nepal Paediatric Society. 2015;35(1):94-95.

17. Mullassery D, Turnock R, Kokai G. Perineal groove. J Pediatr Surg. 2006;41(3):e41-43.

18. Verma SB, Wollina U. Perineal groove-a case report. Pediatr Dermatol. 2010;27(6):626-627.

19. Diaz L, Levy ML, Kalajian A, Metry D. Perineal groove: a report of 2 cases. JAMA Dermatol. 2014;150(1):101102.

20. Halvorson EE, G.M., KUMTA S, KATZ C, AJL S. Two cases of perianal redness. 2012. cited 2019; Available from: https://www.consultant360.com/articles/two-casesperianal-redness.

21. Senanayake SM, Tennakoon UA. Perineal groove leading to a suspicion of child sexual abuse. Ceylon Med J. 2014;59(4):147-148.

22. Wojciechowski M, Van Mechelen K, Van Laere D. Congenital perineal groove. Arch Dis Child. 2019;104(3):286.

23. Esposito C, Giurin I, Savanelli A, Alicchio F, Settimi A. Current trends in the management of pediatric patients with perineal groove. J Pediatr Adolesc Gynecol. 2011;24(5):263-265.

24. Committee on Practice Bulletins-Obstetrics, C.o.G., and Society for Maternal-Fetal Medicine. Practice Bulletin No. 163: screening for fetal aneuploidy. Obstet Gynecol. 2016;127(5):e123-137.

25. Smith GC, Shah I, Crossley JA, Aitken DA, Pell JP, Nelson SM, Cameron AD, et al. Pregnancy-associated plasma protein A and alpha-fetoprotein and prediction of adverse perinatal outcome. Obstet Gynecol. 2006;107(1):161166.

26. Dugoff L, Society for Maternal-Fetal M. First- and second-trimester maternal serum markers for aneuploidy and adverse obstetric outcomes. Obstet Gynecol. 2010;115(5):1052-1061.

27. Yaron Y, Cherry M, Kramer RL, O'Brien JE, Hallak M, Johnson MP, Evans MI. Second-trimester maternal serum marker screening: maternal serum alpha-fetoprotein, beta-human chorionic gonadotropin, estriol, and their various combinations as predictors of pregnancy outcome. Am J Obstet Gynecol. 1999;181(4):968-974.

28. Schieving JH, de Vries M, van Vugt JM, Weemaes C, van Deuren M, Nicolai J, Wevers RA, et al. Alpha-fetoprotein, a fascinating protein and biomarker in neurology. Eur J Paediatr Neurol. 2014;18(3):243-248.

29. Waller DK, Lustig LS, Smith AH, Hook EB. Alpha-fetoprotein: a biomarker for pregnancy outcome. Epidemiology. 1993;4(5):471-476.

30. Chandra S, Scott H, Dodds L, Watts C, Blight C, Van 
Den Hof M. Unexplained elevated maternal serum alpha-fetoprotein and/or human chorionic gonadotropin and the risk of adverse outcomes. Am J Obstet Gynecol. 2003;189(3):775-781.

31. Los FJ, De Bruijn HW, van Beek Calkoen-Carpay T, Hu- isjes HJ. AFP transport across the fetal membranes in the human. Prenat Diagn. 1985;5(4):277-281.

32. Committee on Practice Bulletins-Obstetrics. Practice Bulletin No. 187: neural tube defects. Obstet Gynecol. 2017;130(6):e279-e290. 